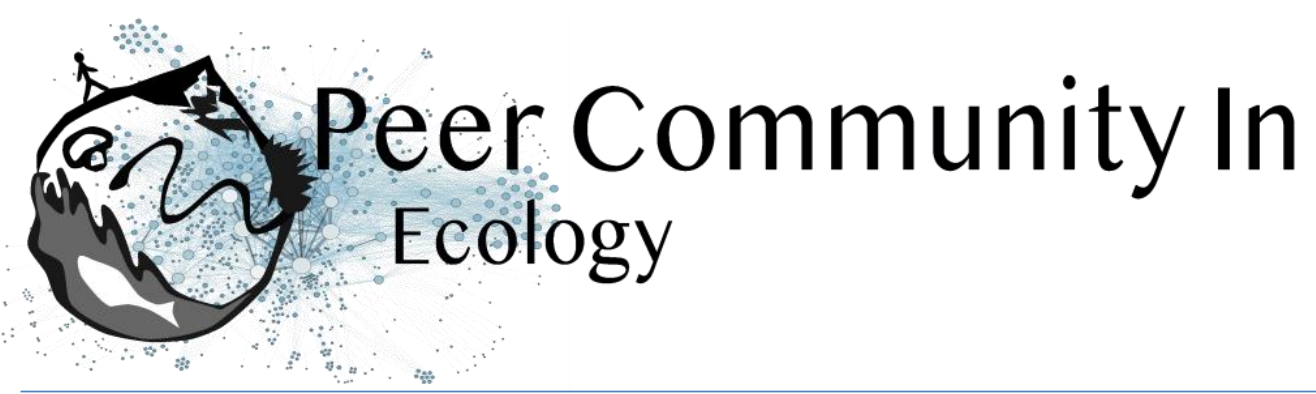

\title{
Assessing bat-vehicle collision risks using acoustic 3D tracking
}

\author{
Gloriana Chaverri based on reviews by Mark Brigham and Brock \\ Fenton
}

\section{A recommendation of:}

Charlotte Roemer, Aurélie Coulon, Thierry Disca, and Yves Bas. Influence of local landscape and time of year on bat-road collision risks (2020), bioRxiv, 2020.07.15.204115, ver. 3 peer-reviewed and recommended by Peer Community in Ecology. 10.1101/2020.07.15.204115

\section{Open Access}

Submitted: 20 July 2020, Recommended: 21 December 2020

Cite this recommendation as:

Gloriana Chaverri (2020) Assessing bat-vehicle collision risks using acoustic 3D tracking . Peer Community in Ecology, 100068. 10.24072/pci.ecology.100068

Published: 21 December 2020

Copyright: This work is licensed under the Creative Commons Attribution-NoDerivatives 4.0 International License. To view a copy of this license, visit http://creativecommons.org/licen ses/by-nd/4.0/
The loss of biodiversity is an issue of great concern, especially if the extinction of species or the loss of a large number of individuals within populations results in a loss of critical ecosystem services. We know that the most important threat to most species is habitat loss and degradation (Keil et al., 2015; Pimm et al., 2014); the latter can be caused by multiple anthropogenic activities, including pollution, introduction of invasive species and fragmentation (Brook et al., 2008; Scanes, 2018). Roads are a major cause of habitat fragmentation, isolating previously connected populations and being a direct source of mortality for animals that attempt to cross them (Spellberg, 1998).

While most studies have focused on the effect of roads on larger mammals (Bartonička et al., 2018; Litvaitis and Tash, 2008), in recent years many researchers have grown increasingly concerned about the risk of collision between bats and vehicles (Fensome and Mathews, 2016). For example, a recent publication by Medinas et al. (2021) found 509 bat casualties along a 51-km-long transect during a period of 3 years. Their study provides extremely valuable information to asses which factors primarily drive bat mortality on roads, yet it required a substantial investment of time coupled with the difficulty of detecting bat carcasses. Other studies have used acoustic monitoring as a proxy to gauge risk of collision based on estimates of bat density along roads (reviewed in Fensome and Mathews 2016); while the results of such studies are valuable, the number of passes recorded does not necessarily equal collision risk, as many species may simply avoid crossing the roads. Understanding the risk of collisions is of vital importance for adequate planning of road construction, particularly for key sites that harbor threatened bat species or unusually large populations, especially if these are already greatly impacted by other anthropogenic activities (e.g. wind turbines; Kunz et al. 2007) or unusually deadly pathogens (e.g. white-nose syndrome; Blehert et al. 2009).

The study by Roemer et al. (2020) titled "Influence of local landscape and time of year on bat-road collision risks", is a welcome addition to our understanding of bat collision risk 
as it employs a more accurate assessment of bat collision risk based on acoustic monitoring and tracking of flight paths. The goal of the study of Roemer and collaborators, which was conducted at 66 study sites in the Mediterranean region, is to provide an assessment of collision risk based on bat activity near roads. They collected a substantial amount of information for several species: more than 30,000 estimated flight trajectories for 21+ species, including Barbastella barbastellus, Myotis spp., Plecotus sp., Rhinolophus ferrumequinum, Miniopterus schreibersii, Pipistrellus spp., Nyctalus leisleri, and others. They assess risk based on estimates of 1) species abundance from acoustic monitoring, 2) direction of flight paths along roads, and 3) bat-vehicle co-occurrence.

Their findings suggest that risk is habitat, species, guild, and season-specific. Roads within forested habitats posed the largest threats for most species, particularly since most flights within these habitats occurred at the zone of collision risk. They also found that bats typically fly parallel to the road axis regardless of habitat type, which they argue supports the idea that bats may use roads as corridors. The results of their study, as expected, also show that the majority of bat passes were detected during summer or autumn, depending on species, yet they provide novel findings of an increase in risky behaviors during autumn, when the number of passes at the zone of collision risk increased significantly. Their results also suggest that mid-range echolocators, a classification that is based on call design and parameters (Frey-Ehrenbold et al., 2013), had a larger portion of flights in the zone at risk, thus potentially making them more susceptible than short and long-range echolocators to collisions with vehicles.

The methods employed by Roemer et al. (2020) could further help us determine how roads pose species and site-specific threats in a diversity of places without the need to invest a significant amount of time locating bat carcasses. Their findings are also important as they could provide valuable information for deciding where new roads should be constructed, particularly if the most vulnerable species are abundant, perhaps due to the presence of important roost sites. They also show how habitats near larger roads could increase threats, providing an important first step for recommendations regarding road construction and maintenance. As pointed out by one reviewer, one possible limitation of the study is that the results are not supported by the identification of carcasses. For example, does an increase in the number of identified flights at the zone of risk really translate into an increase in the number of collisions? Regardless of the latter, the paper's methods and results are very valuable and provide an important step towards developing additional tools to assess bat-vehicle collision risks.

\section{References}

[1] Bartonička T, Andrášik R, Dul’a M, Sedoník J, Bíl M (2018) Identification of local factors causing clustering of animal-vehicle collisions. The Journal of Wildlife Management, 82, 940-

947. https://doi.org/10.1002/jwmg.21467

[2] Blehert DS, Hicks AC, Behr M, Meteyer CU, Berlowski-Zier BM, Buckles EL, Coleman JTH, Darling SR, Gargas A, Niver R, Okoniewski JC, Rudd RJ, Stone WB (2009) Bat White-Nose Syndrome: An Emerging Fungal Pathogen? Science, 323, 227-227. https://doi.org/10.1126/science.1163874

[3] Brook BW, Sodhi NS, Bradshaw CJA (2008) Synergies among extinction drivers under global change.

Trends in Ecology \& Evolution, 23, 453-460. https://doi.org/10.1016/j.tree.2008.03.011

[4] Fensome AG, Mathews F (2016) Roads and bats: a meta-analysis and review of the evidence on vehicle collisions and barrier effects. Mammal Review, 46, 311-323. https://doi.org/10.1111/mam.12072

[5] Frey-Ehrenbold A, Bontadina F, Arlettaz R, Obrist MK (2013) Landscape connectivity, habitat structure and activity of bat guilds in farmland-dominated matrices. Journal of Applied Ecology, 50, 252-

261. https://doi.org/10.1111/1365-2664.12034

[6] Keil P, Storch D, Jetz W (2015) On the decline of biodiversity due to area loss. Nature Communications, 6 , 8837. https://doi.org/10.1038/ncomms9837

[7] Kunz TH, Arnett EB, Erickson WP, Hoar AR, Johnson GD, Larkin RP, Strickland MD, Thresher RW, Tuttle MD (2007) Ecological impacts of wind energy development on bats: questions, research needs, and hypotheses. Frontiers in Ecology and the Environment, 5, 315-324. https://doi.org/10.1890/1540- 
Management, 42, 688-697. https://doi.org/10.1007/s00267-008-9108-4

[9] Medinas D, Marques JT, Costa P, Santos S, Rebelo H, Barbosa AM, Mira A (2021) Spatiotemporal persistence of bat roadkill hotspots in response to dynamics of habitat suitability and activity patterns. Journal of Environmental Management, 277, 111412. https://doi.org/10.1016/j.jenvman.2020.111412 [10] Pimm SL, Jenkins CN, Abell R, Brooks TM, Gittleman JL, Joppa LN, Raven PH, Roberts CM, Sexton JO (2014) The biodiversity of species and their rates of extinction, distribution, and protection. Science, 344. https://doi.org/10.1126/science.1246752

[11] Roemer C, Coulon A, Disca T, Bas Y (2020) Influence of local landscape and time of year on bat-road collision risks. bioRxiv, 2020.07.15.204115, ver. 3 peer-reviewed and recommended by Peer Community in Ecology. https://doi.org/10.1101/2020.07.15.204115

[12] Scanes CG (2018) Chapter 19 - Human Activity and Habitat Loss: Destruction, Fragmentation, and Degradation. In: Animals and Human Society (eds Scanes CG, Toukhsati SR), pp. 451-482. Academic Press. https://doi.org/10.1016/B978-0-12-805247-1.00026-5

[13] Spellerberg I (1998) Ecological effects of roads and traffic: a literature review. Global Ecology \& Biogeography Letters, 7, 317-333. https://doi.org/10.1046/j.1466-822x.1998.00308.x

\section{Revision round \#2}

2020-10-30

Dear Charlotte and co-authors,

I thank you for making the changes suggested by the reviewers on your preprint titled "Influence of local landscape and time of year on bat-road collision risks". I believe you have adequately addressed all of our suggestions; however, new ones have arisen from your changes and from a more thorough revision of the entire text. For this new version I decided not to send it out for review again, but would like you to carefully consider my following suggestions before I give the preprint my recommendation.

Line 20: remove moreover. Line 23: Remove "bats avoiding vehicles or not" from the text, and keep it to what you can measure (bat-car co-occurrence). Line 42: change maximal to greatest. Lines 42-43: this sentence is confusing, as it seems that there might only be these two options. Do you mean that bats followed the road axis regardless of the type of habitat present? Line 57: change enhances to increases (remove increasing at the beginning of this line). Line 58: put comma after parenthesis. Lines 58-59: What do you mean by "results concerning the role of traffic or speed limit on road-kills were CONTRASTED for other vertebrate taxa"? Lines 60-62: Please modify this sentence. My suggestion is: Studies on a variety of animal groups also found that preferred habitats for foraging or movement, described at the home-range scale (e.g. presence or absence of woodland, cropland, wetland ...), are more often... Line 64: I think here you are referring to habitat rather than landscape features. Line 76: Do you mean here that gaps in vegetation are an important factor that is known to increase road collisions? Line 93: Change qualified to classified. Line 101: Add road here, for example "decreases bat road crossing". Lines 107-109: You need a reference to support this claim. Doing this here is very important since you are not providing any evidence in the introduction, or elsewhere, that flight direction is relevant for understanding collision risk. Lines 121-125: This last part does not seem to be adding anything to the main topic of this paragraph, so l'd suggest you remove it. Lines 129133: I would recommend you summarize this part. My suggestion: "The aim of our study is to assess the effects of the local habitat, coupled with bat density and movement patterns, on road collision risks." Right now it's too long and fairly repetitive, since some of the specific methods mentioned in this section are basically tackling the same goals. Also, note that I've changed landscape to habitat, since you are looking at small-scale, not large-scale, features. Lines 140-143: I am not sure you have provided sufficient evidence as to why you'd expect bat density or the proportion of animals flying in the zone at collision risk to influence collision risk in different ways in different contexts. I think the ideal approach is to simply mention that it's expected that larger number of bats flying in the risky zone would mean greater collision risks. If not, then there may be site, habitat and/or species-specific traits that influence collision risks. Lines 143-150: I think it 
is now great that you have added some predictions, but in my opinion, they still need a lot of work. Let's take it one by one: (1) a higher bat density at good quality habitats (i.e. tree rows near streams and tall trees) and at roads with a lower traffic rate. These are two separate predictions, right? Also, is it possible to generalize that having tree rows near streams and tall tress is a good habitat for all species considered? One alternative here is to simply mention that previous studies have found greater activity in sites with those type of conditions, similarly to what's mentioned in lines 92-94. Provide some evidence (perhaps a reference) that can support the second part of this prediction (more cars $=$ fewer bats). Your second prediction, (2) a higher proportion of individuals in the zone at collision risk when vegetation grows closer to the road and in habitats with dense vegetation at each side of the road compared to habitats without trees. Are these two, again, separate predictions since they represent different explanatory variables? For your third prediction, (3) a correlation between the orientation of bat trajectories and the orientation of linear vegetation, you need a reference to support this assertion. If there are no previous studies that provide this evidence, then you need to remove this from your predictions. Overall, I don't understand the need to separate your predictions by response variable. For example, why expect that traffic affects bat density but not flying in the zone at collision risk, or that habitat configuration affects flying in the zone at collision risk but not bat density? Line 172: Abbreviate genus name upon second mention (e.g. Pinus halepensis). Table 1 (legend): What is the second sentence referring to? Line 198: Change cumulated to accumulated. Line 263: I don't understand why you mention Figure 2 here. I would suggest to remove this. Figure 3: Why is the estimation of flight trajectory orientation a qualitative component of your model? I am still not entirely convinced that this estimate, flight trajectory, is adding much to our understanding of collision risk, primarily because you have not provided evidence from previous studies that either a parallel or perpendicular trajectory entails greater risks. Line 314: Change "to fly" to flying. Line 315: Is this estimate including flights only at a vehicle height or does this include at vehicle height on the road? Lines 326-328: I am still not convinced that you can safely assume this (still seems speculative), so I recommend that you delete this sentence. Lines 329-332: This is still something that must be addressed carefully, mainly in terms of providing evidence that a parallel, or perpendicular, flight path orientation entails greater collision risks. Lines 362-363: Can you please explain this a bit better? I am not sure first why you did this, or how. Why do you need your explanatory variables to follow a normal distribution? How did you normalize them? Line 364: remove the error message. Line 394: Change problem to problems. Lines 394-397: What was done with the models that did not converge? Legend figure 4: Please arrange the order of the $x$-axis categories in the same order as those displayed in the graphs (e.g. first $F$, then FE, DPT, etc.). Line 401: Landscape (habitat?) type refers to which variables in table 2? Perhaps what is needed is a new main column in table 2 called habitat/landscape type and then subdivide it with pertinent categories. Table 2: Please switch columns and rows. Your columns should be the explanatory variables, and rows should be species. The same applies to tables 3 to 5. Line 442: I believe some results should be provided to understand this a bit better, so please remove "results not shown" and provide more evidence for your assertion. At the moment the only way to understand flight trajectories is to look at the estimate of the model in table 5, which is not that straightforward. Lines 457-460: It is normally not widely accepted that paragraphs are composed of a single sentence. In this case, I believe these two ideas should be merged into a single paragraph. Line 500: change to explanatory variables. Lines 507-508: you need a reference here. Lines 518-522: My opinion is that you do not need to explain all of your results, especially if there is no way to explain them without resorting to speculation. For example, you mention that there is higher social activity in N. leisleri, but you have provided no evidence for this. I'd suggest you remove this entire sentence. Line 547: The word "avoided" implies that bats actively reduce their activity in the zone at risk when they perceive traffic, which you are not providing evidence for. I also do not agree with lines 550-551: "These results show that bats spatially avoid the vicinity of vehicles". I guess you'd need a similar set of data as that obtained by Zurcher et al. (2010), where you can clearly see that bats change their course when cars are detected, to explain a reduction of a species' activity at the zone at collision risk during higher traffic. Some of the speculation in this paragraph continues in the next one, particularly in line 558 ("more reluctant to approach vehicles") and line 563 ("their vehicle avoidance behaviour"). Lines 567-575: You are focusing this section on the more speculative ideas rather than those for which you might have greater support from previous studies (and your own data). Why is there an increase in flights at the zone of risk during the summer, for example? You did not explain this in the discussion. This increase may simply be the result of an increase in bat density 
during the summer (which you clearly show in your data), and not necessarily because there is a larger proportion of those flights occurring in the zone at risk (implying riskier behaviors or greater naïveté). The only two species for which I believe you have sufficient evidence of an increase in the number of bat passes at risk per night as the years progresses (and greater during the autumn compared to the summer) are $P$. pygmaeus and Plecotus sp. For those two species, the number of bat passes at risk per night may increase due to an overall increase in bat density and/or a larger proportion of flights occurring at the zone of risk. Without additional data, such as whether those flights at risk are predominantly juvenile, or evidence from other species which also have volant juveniles during autumn, the section about juveniles and sexual activity driving risky behaviors is speculative. Lines 627-628: I believe the aims of your study can be attained (at least approximated) with direct counts of carcasses, yet with a significantly greater investment of time. Maybe this would be worth mentioning, adding a reference to studies that have counted carcasses to asses risk (e.g. Medinas et al. 2020: https://doi.org/10.1016/j.jenvman.2020.111412). Lines 633-635: add a reference to this sentence. Line 651: change to "habitat loss for numerous". Lines 665-666: Remove authors from parenthesis.

I thank you for your continuous effort to improve this wonderful preprint, and hope to see it finalized soon.

Kind regards,

Gloriana Chaverri

Preprint DOI: $10.1101 / 2020.07 .15 .204115$

\section{Author's reply:}

Please find our answer in the attached document.

Download author's reply (PDF file)

\section{Revision round \#1}

2020-08-20

Dear Charlotte and co-authors,

We have already received comments (see below) on your preprint titled "Influence of local landscape and time of year on bat-road collision risks". Overall, the reviewers seem enthusiastic about your work, as am I, but point to a few issues that hopefully you can address before I decide to recommend the preprint. As you will notice, in addition to the comments by Brock Fenton and Mark Brigham, two of Mark's students kindly helped in the evaluation process and I am sure you will find their suggestions useful.

I also have a few comments of my own. First, I agree with Mark in that there is some speculation in the discussion that should be avoided. Some examples where I see speculation in the discussion: lines 487-491, 514, 519-520, 524-526, 535-537.

Second, I am also having some difficulty with a few of your response variables and their contribution to your main question: what factors increase collision risks in bats? At the moment the only variable I think really would help you answer your question is the number of bat passes at collision risk per night. At the moment you are including in your model 1) number of bat passes per night, which does not directly measure risk. You also include the 2) proportion of bat flights at vehicle height, yet this will not provide you with a total risk estimate. For example, in site A say you only detect two passes in a given night and both are at vehicle height, but in site $B$ you detect 100 passes and 50 are at vehicle height; with these data you will find that $100 \%$ of passes are at risk at site $A$ and only $50 \%$ at site $B$, when clearly more animals are at risk in site $B$. This will have major implications if and when proposing strategies to reduce vehicle collisions with bats. The other response variable, 3 ) vehicle avoidance, is very confusing in my opinion and assumes that bats are actively avoiding vehicles, which you are not providing strong evidence for. So to recapitulate, my suggestion is that a 


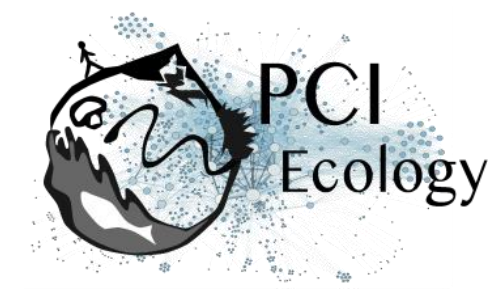

much more straightforward and clean model would only need to include bat passes at collision risk per night as your response variable. I see no need to include trajectory orientation since your variable measuring collision risk already takes into account the fact that bats are entering the zone of risk (above the road and at vehicle height).

Finally, I would like to congratulate you on this wonderful work and all the effort undertaken to provide such detailed information on this very important topic.

Gloriana Chaverri

Additional requirements of the managing board:

As indicated in the 'How does it work?' section and in the code of conduct, please make sure that:

-Data are available to readers, either in the text or through an open data repository such as Zenodo (free), Dryad or some other institutional repository. Data must be reusable, thus metadata or accompanying text must carefully describe the data.

-Details on quantitative analyses (e.g., data treatment and statistical scripts in $\mathrm{R}$, bioinformatic pipeline scripts, etc.) and details concerning simulations (scripts, codes) are available to readers in the text, as appendices, or through an open data repository, such as Zenodo, Dryad or some other institutional repository. The scripts or codes must be carefully described so that they can be reused.

-Details on experimental procedures are available to readers in the text or as appendices.

-Authors have no financial conflict of interest relating to the article. The article must contain a "Conflict of interest disclosure" paragraph before the reference section containing this sentence: "The authors of this preprint declare that they have no financial conflict of interest with the content of this article." If appropriate, this disclosure may be completed by a sentence indicating that some of the authors are $\mathrm{PCl}$ recommenders: "XXX is one of the $\mathrm{PCI} X X X$ recommenders."

Preprint DOI: $10.1101 / 2020.07 .15 .204115 \mathrm{v} 1$

\section{Reviewed by Brock Fenton, 2020-07-21 20:40}

This is an interesting and timely topic ... but there are some missing elements.

The first missing element is a clear statement of the hypothesis underlying the work, and, associated with this, specific predictions that can be tested with the data. This approach would help the reader better appreciate the importance of the paper.

More disturbing is the absence of any data about bat mortality during the study. Were any road-killed bats found? Did the authors look for road-killed bats?

It is possible that a skillfully constructed hypothesis and predictions would circumvent the lack of data on mortality, but I am not sure how. I realize that the absence of road-killed bats would not conclusively show no mortality, as bats are small and might not last long on the road.

Another important issue could be the number of known bat roosts in the study area. But hindsight is a wonderful tool.

How would the findings of this paper alter conservation actions designed to protect bats?

I like the idea of the study but without evidence in the form of road-killed bats, I find it difficult to fully appreciate the work.

Brock Fenton

Reviewed by Mark Brigham, 2020-08-18 21:04 
Overall. I think the premise of this work is very thoughtful and useful. While I am no expert about the analysis undertaken, it largely makes sense to me and I think the 3-D analysis is highly thoughtful. It's a shame that bats all react differently to vegetation, traffic volume etc. and there is no general outcome that applies even to most species. But that is what the science says and so be it.

Writing. I think the manuscript would benefit greatly from a thorough edit by a native English speaker. There are multiple instances of tense-subject and verb-subject disagreement. There are also numerous instances of awkward sentence construction that reflect French rather than English grammar. In some instances, this makes it difficult to ascertain the meaning of sentences so it is more than just a bit of a nuisance. I recognize that it must be inordinately difficult to write in a language other than one's native tongue and it is totally unfair that English is the language of science, but it is what it is.

Another thing that would help re writing would be to reduce the use of acronyms which makes reading harder. Not sure they are all really necessary (e.g., TOAD).

Sample sizes. The number of nights of sampling is reported differently in the manuscript. The Abstract says 2 nights whereas in the body of the text of the Methods it is reported as 2-5 nights. We are not told when the sampling happened and thus how there is the ability to assess the seasonal differences that are reported. Further you say that sampling was done on "straight portions" of roads. Define what a straight portion is more precisely. Rather than defining sampling as occurring during optimal weather conditions, tell us what the min and/or max temperatures, maximum wind speed and maximum amount of precipitation were. Likewise for distance between microphones; give us the absolute max and min rather than the variation.

Actual collisions. In my view it needs to be made much more explicitly clear in the Introduction that vehiclebat collisions are indeed common. This is buried amongst many citations to non-bat animals. While I think making the rationale of broad interest is beneficial, deer are not bats. It was my impression based on many years of driving at night, that bats flying over top of roads are very uncommon. I think that in all the night driving I have done I have only ever hit one and it was unhurt (An unusual technique for catching bats. Bat Research News 1996 37:115). However, the authors cite a number of papers that I was unaware of which show that my impression was wrong. I do think many others would share my impression. I think it is well known that bat flight is affected by roads and this may cloud the impression of many. I would disagree that it has not been emphasized that bats follow roads; I think this is reasonably well-established in the literature.

3-D positioning. This analysis is most interesting and I found it really impressive how you did this. I think you were appropriately conservative in assigning a position to the bats. I thank you for providing access to the $\mathrm{R}$ script. The precise definition of safe vs. unsafe was very clear. Well done. I like that you actually calculate an estimate of how many recorded passes actually put a bat into an unsafe position and thus estimate the actual number of potential collisions. While it obviously would be an overestimate, it gives some information about how many bats really could be killed by vehicles. However, this outcome does not get emphasized enough in the Discussion. I know it is impossible except at a species-specific level but some direct indication of what proportion of passes puts bats at risk would be useful.

Outcomes. I find it interesting that the factors you assessed affect different species in really different ways so there appears to be no one thing that can be done to reduce the risk to bats. Line 514 - not sure it is appropriate to assume that some bats "recognize the danger triggered by vehicles" (this is also an instance where the writing needs to be clearer - I think you mean to say "recognize the risk posed by vehicles"). To test this would take a specific data set. Line 521 - if higher traffic volume is trivial, why talk about it? Overall in the Discussion I think there is too much speculation - e.g., warm asphalt effects, Rhinolophus issues. Stick to the main outcomes and add a real estimate of likelihood of collision. Do you really think that forests around roads should be cleared to reduce risk to bats? It appears to me that this would really only help some.

Tables and Figures. I am not sure that the inset to Figure 1 is really necessary. All of the sampling sites are in the same biogeographic region - so just say that. Tables 2-4 are very busy. I don't have any good suggestions about how to make them better but there is a lot to take in. 


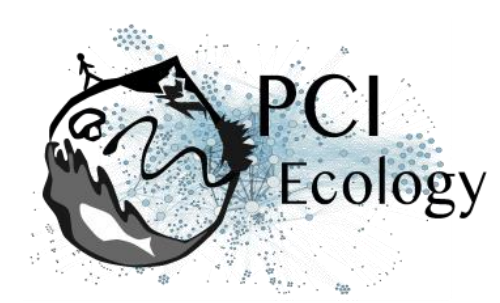

Mark Brigham

I also had two of my students undertake independent reviews of the MS. Those reviews follow.

Student 1.

Overall, this study was well-conducted and contained interesting and valid data, particularly the threedimensional call modeling. However, the main issue lies in a lack of clarity and cohesion in the writing. With this in mind, I suggest Roemer et al. make the following changes to their manuscript:

- Provide further justification for the study other than "no study provides an understanding" of the topic thus far (line 19).

- Include examples of the "many questions [that] remain concerning how local landscape features may influence bat behaviour" (line 18).

- Address the discrepancy in the number of nights per site (line 128) and why visible moon percentage was not considered a criterion (line 165).

- Provide evidence to support the claim that "driver avoidance of collision is probably less important for smaller animals" (line 65).

- Define "tree essences" and how they differ (line 475).

- Address issues of clarity and brevity in the writing. Certain phrases fail to translate well into English and result in disjointed sentences, such as " $\mathrm{P}$. kuhlii/nathusii avoided more the zone at collision risk when traffic increased..." (lines 513-514) and "probably because bats have no choice but crossing the road closely in time to vehicle passes when traffic is high" (lines 522-523). Other issues with word order include "zone at collision risk" (lines 32-33) as opposed to "collision risk zone". In addition, there are several instances of verbose writing that would benefit from re-wording, such as "our study aimed at 1) assessing the effects of the characteristics of the local landscape on bat activity and movement behaviour and consequently on road collision risks" (lines 109-110).

- Verify that verb tenses are being used consistently, such as "local landscapes eliciting the highest proportion of flights at collision risk for this species were forests" (line 478).

- Proofread for typos prior to submission, such as missing conjunctions (line 47) and "a lesser extent" instead of "a lesser extend" (line 566).

Student 2.

I want to begin by saying I am no expert on this topic and am just beginning my career in the field of scientific research but I hope my comments can help.

In my opinion, you collected an extensive amount of good data that indicates differences in the collision risks of bats based on a variety of variables. However, the quality of the study is undermined by lack of clarity and so much information that understanding and following the manuscript proved rather difficult.

In the abstract it is stated that sites were sampled for two full nights; in the methods it is indicated that sites were sampled for two to five nights; and ultimately the effect of seasonality on bat collision risks was investigated. The sampling schedule needs to be clarified and explained so readers can understand exactly how often and for how long sites were sampled. For example, if sites were sampled for only "two full nights", how can seasonality be considered in the study? My guess is that you sampled sites for two to five nights with the total amount of time over those nights amounting to two full nights?

I envy your efforts, the amount of data you collected, and the number of variables you considered; however, ultimately I think including this large a breadth of information all in one manuscript was not beneficial. There are simply too many variables and so much information covered that I had great difficulty following and understanding what the take home message was meant to be. Perhaps focusing on the local tree height and 


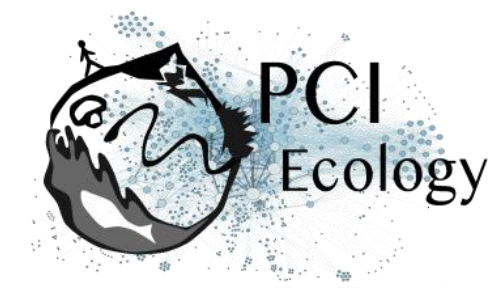

distance from the road and how they may influence the flight position of bats over roads would clear up the writing and improve the focus. I think a lot of really interesting information is lost amongst the large amount of text and I hope you consider revisiting the results section in particular to see if there is a clearer way of conveying your findings.

There are a few occurrences of typos and poor grammar and I unfortunately cannot send a marked-up version of the manuscript because I could not edit the document but I do encourage a review of the manuscript to look at the sentence and grammar structure.

I applaud you for revealing the potential conflict of interest of Charlotte Roemer and Thierry Disca. I believe that is really respectable and good on you.

\section{Author's reply:}

Please read our answer to the reviewers and the new version of our manuscript in the attached documents. 